\title{
WAVE PROPAGATION AND BAND-GAP CHARACTERISTICS OF CHIRAL LATTICES
}

\author{
Stefano Gonella, Alessandro Spadoni, Massimo Ruzzene* \\ School of Aerospace Engineering \\ Georgia Institute of Technology \\ Atlanta, Georgia 30332
}

\author{
Fabrizio Scarpa \\ Department of Mechanical Engineering \\ University of Bristol \\ Bristol, UK
}

\begin{abstract}
Plane wave propagation in a chiral lattice is investigated through the application of Bloch's theorem. Two-dimensional dispersion relations are estimated and analyzed to illustrate peculiar properties of chiral or non-centrosymmetric configurations and investigate the directional behavior of wave propagation for varying geometric parameters. Special attention is devoted to the determination of phase and group wave velocities. Considerations based upon the analysis of velocity plots are compared with results obtained from dispersion curves to further validate the directional behavior of the proposed lattice. Finally, The relation of directionality with frequency of the traveling waves is discussed.
\end{abstract}

\section{INTRODUCTION}

Honeycombs are multifunctional structural assemblies that have generated significant improvements over solid material components; high strength-to-weight ratio, thermal and acoustic insulation capabilities as well as design flexibility are among some of the excellent characteristics of honeycombs [1]. Exhaustive investigations have established the advantages offered by such structural arrangements and their substantial employment in the aerospace industry is now broadening to more technologically conservative industries.

More recently, structural honeycombs have become the focus of studies concerned with the characteristics of wave propagation though periodic media, in a quest to uncover systems that can be tuned to control the transmission of mechanical energy.
In periodic structures, the impedance mismatch generated by periodic discontinuities in the geometry, acting as a waveguide, and/or in the constituent material, causes destructive wave interference phenomena over specific frequency bands called "stopbands" or "bandgaps". In 2-D periodic structures, the location and the extent of bandgaps depend on the nature of wave propagation [2]. The directional behavior of energy propagation complements bandgap characteristics of all periodic assemblies, and suggests their application as directional mechanical filters. To this end, research has been conducted to relate specific unit cell configurations to particular behaviors in order to design lattices with given vibration isolation characteristics [3]. Wave propagation in a hexagonal honeycomb, one of the most common lattice structures, has recently been investigated by [4]. Other recent studies [5,6] have uncovered the directional characteristics ("beaming effects") of cylindrical grids as well as hexagonal and re-entrant 2-D lattices.

A very novel subclass of periodic structural systems is that of "auxetic" lattices, which feature a negative in-plane Poisson's ratio [7]. According to [7], the unusual negative Poisson's ratio in cellular solids, in the form of polymers, metal foams, honeycombs, and even rocks, can be attributed to three aspects: the presence of rotational degrees of freedom, non-affine deformation kinematics, or anisotropy. Rotational degrees of freedom stem from the presence of non-central forces, which must be accompanied by a moment to satisfy equilibrium equations. Nonaffine deformations arise whenever internal forces do not depend on distance but instead only connect selected pairs of particles, which move apart during stretching while others do not. 
The last aspect proposed by [7] is that of anisotropy or noncentrosymmetry, which defines a structure which is not equivalent to its mirror image, thus lacking a center of symmetry. Such structural systems are also termed chiral structures.

The focus of the current work is to determine the stop-band behavior of a novel auxetic assembly, named chiral honeycomb, proposed by [8]. The chiral honeycomb is an example of noncentrosymmetric (or chiral) as well as auxetic assemblies, owing the lack of a center of symmetry, and a negative, in-plane Poisson's ratio [8]. Chiral effects, however, cannot be expressed within classical elasticity as the modulus tensor- in the form of compliance or stiffness tensors both under plane stress and plane strain assumptions- required to describe chirality would have to be of odd rank, differently from that of classical elasticity theory [9]. Chiral assemblies hence, if represented by an equivalent homogeneous model, require higher order elasticity theories to account for micro-rotations within the material such as micropolar continuum or Cosserat elasticity theory [9]. Chirality, yet, has no effect on the classical engineering constants of an equivalent homogeneous material [9]; subsequently, the microscopic behavior of the chiral honeycomb is expressed by a finite-element discretization of the constituent frame-like structure. Chiral lattices, although not amenable to classical elastic theories, are periodic structures, and as such can be analyzed with Bloch's theorem [10], which establishes conditions for the analysis of wave propagation in a unit cell, characteristic of an infinite assembly. Bloch's theorem is based merely on the spatial periodicity of a system, and thus, should not be limited by constraints imposed by chirality. Investigations are carried out in the reciprocal lattice space, defined by a vector basis describing the symmetry directions of the chosen unit cell. The analysis is further reduced by only considering irreducible Brillouin zones introduced by the landmark work in solid state physics by [11]. Engineering applications of such techniques can be found in the extensive literature by Mead et al. [10]. Special attention is also devoted to the determination of phase and group velocities. A combined inspection of velocity plots and phase constant surfaces is carried out to show the dependence of the directional behavior of lattices upon the geometry of the unit cell and the frequency of wave propagation.

The remainder of this paper is organized in 5 sections. This introduction is followed by the motivations that lead to the results presented here. Secondly, a description of the chiral honeycomb and its mechanical properties is given. The analysis of the free wave motion is planar lattices is described in detail, followed by a discussion of the obtained results. A summary of the findings and recommendations for future work are lastly offered.

\section{OBJECTIVES}

A 2-D chiral lattice is an example of systems that are not invariant to coordinate inversion, and thus a corresponding con- tinuum representation cannot be expressed by classical elasticity theory [9]. The stiffness or compliance tensor in classical elasticity theory if of fourth-order rank, and as such is unchanged by a coordinate inversion. A continuum representation of chiral lattices then requires generalized continuum elastic theories such as micropolar elasticity [9]. Ongoing research by the authors is devoted to representing just such a generalized elasticity model for the chiral lattice proposed here. In the current framework, on the other hand, in-plane wave propagation characteristics are analyzed solving the eigenvalue problem which arises when boundary conditions are imposed to the homogeneous governing equations of motion, as a Sturm-Liouville problem. Such procedure allows for the analysis of wave propagation in a periodic infinite medium by analyzing a characteristic portion of the lattice, the unit cell, which when repeated along symmetry directions yields the entire lattice domain.

\section{GEOMETRY OF A CHIRAL LATTICE AND ITS MECHAN- ICAL PROPERTIES}

The structural layout of a chiral lattice, shown in Fig. 1, consists of circular elements of radius $r$, acting as nodes, connected by ribs or ligaments, of length $L$ tangent to the nodes themselves. The distance between node centers is denoted as $R$, while the angle between the imaginary line connecting the node centers and the ribs is defined as $\beta$. The angle between adjacent ligaments is denoted as $\theta$. Finally,the wall thickness of nodes and ribs is denoted as $t_{c}$ and $t_{b}$ respectively. As described in [8], the following geometric relationships hold:

$$
\sin \beta=\frac{2 r}{R}, \quad \tan \beta=\frac{2 r}{L}, \quad \sin \theta=\frac{R / 2}{R}, \quad \cos \beta=\frac{L}{R} .
$$

As demonstrated by the third of Eqn. (1), the angle $\theta$ is always $30^{\circ}$, which indicates hexagonal symmetry, yielding a lattice with in-plane mechanical isotropy [8]. Geometrically however, the honeycomb being analyzed possesses two-dimensional chiral symmetry, which yields a lattice not invariant to coordinate inversions [9].

The resulting cellular structure is characterized by the following unique mechanical properties:

Negative in-plane Poisson's ratio: The negative Poisson's ratio (theoretically found to be $\approx-1$ [8]) is the result of a unique deformation mechanism, illustrated by the linearelastic model proposed by [8]. When an in-plane stress is applied to a chiral honeycomb lattice, a torque $\mathrm{M}$ bends the ligaments into a sigmoid shape and produces a rotation of the nodes; meanwhile, the angle $\theta$ remains constant at $30^{\circ}$ as indicated by Eqn. 1. The ligaments remain tangent to 


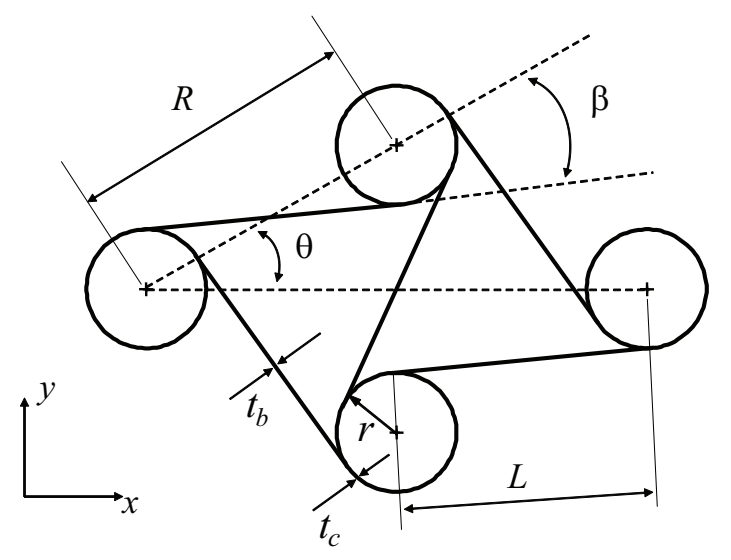

Figure 1. CHIRAL LATTICE GEOMETRY.

the nodes producing a macroscopic deformation which corresponds to a change of area without change of shape. The resulting deformation is that of winding ligaments onto the nodes [8].

High shear modulus: the shear modulus for the considered chiral structure is expected to be very high as a result of the Poisson's ratio being equal, or close to -1 .

High elastic displacement capabilities: the deformation mechanism of the chiral geometry, whereby the ligaments bend and tend to wrap around the nodes can lead to large overall deformations for the assembly, with the strain in each component not exceeding the linear range of the stress-strain relationship. This implies that large deformations can be achieved in a reversible fashion.

Design flexibility: the properties and the behavior of chiral assemblies can be significantly altered through variations in the characteristic parameters $L, R$, and $t$. In particular, the compliance properties and the corresponding behavior of chiral assemblies can be significantly altered through variations in the characteristic parameters that define the geometry. The relation between node radius and rib length can be controlled by varying the ratio $L / R$, as shown in Fig. 2, which possess an upper bound limit of 1 . This in turns affects the angle $\beta$ (Eqn. 1) upon which the in-plane Young's modulus is dependent [8]. The properties of the assembly can thus be tailored to achieve desired characteristics by changing a limited number of geometric parameters. As an example, in the limit of $L / R$ approaching 1 , the in-plane mechanical properties and geometry of the triangular honeycomb are recovered.

The unit cell of the honeycomb depicted in Fig. 2 is highlighted in dashed lines, and it constitutes the structural portion sufficient to analyze the in-plane dynamic behavior, as described in the following section.

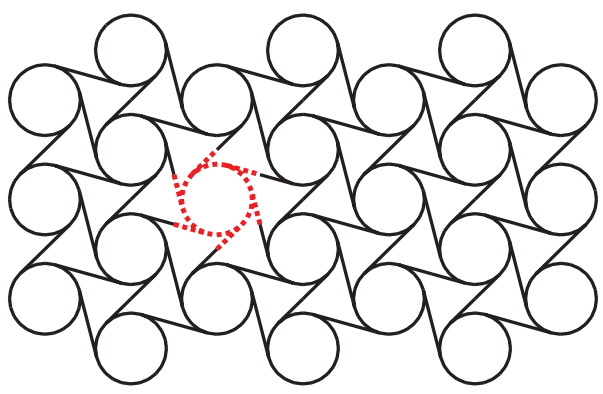

(a)

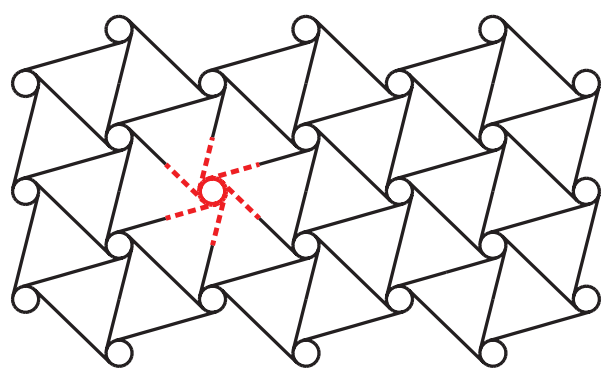

(b)

Figure 2. LATTICE CONFIGURATIONS CORRESPONDING TO $L / R=$ 0.70 (a), AND $L / R=0.97$ (b).

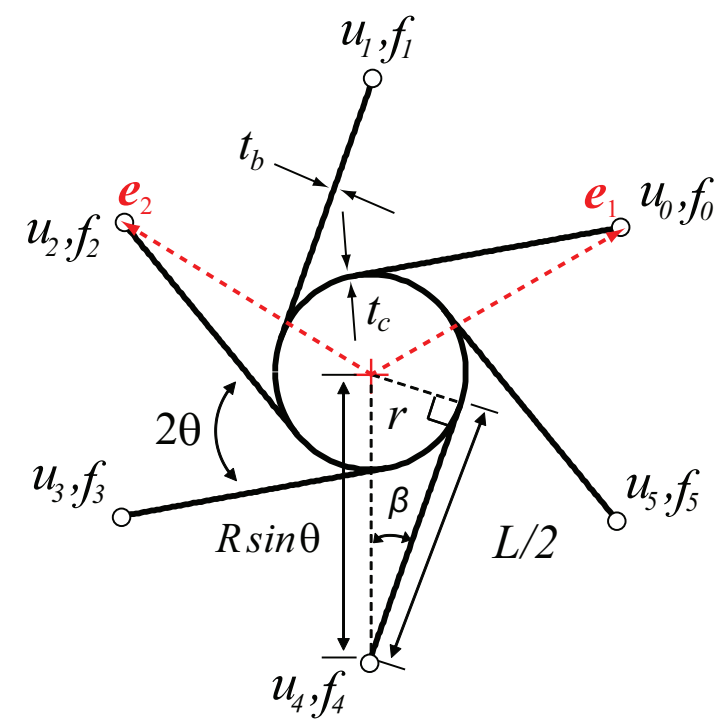

Figure 3. UNIT CELL AND ITS GEOMETRIC PARAMETERS.

\section{UNIT CELL ANALYSIS OF WAVE PROPAGATION IN CHIRAL LATTICES}

The structural lattice under investigation is obtained from the assembly of unit cells of the kind shown in Fig. 3. As in any periodic assembly, the location of a generic point can be de- 
scribed in terms of location within a reference unit cell and a set of lattice vectors which define the periodicity of the assembly. We introduce a reference frame in the plane of the lattice $\mathcal{F}_{I}$ defined by an orthogonal unit vector basis $I=\left(\boldsymbol{i}_{1}, \boldsymbol{i}_{2}\right)$, such that the location of a point $P$ in cell $n_{1}, n_{2}$ can be expressed as:

$$
\boldsymbol{\rho}_{P}\left(n_{1}, n_{2}\right)=\boldsymbol{r}_{P}+n_{1} \boldsymbol{e}_{1}+n_{2} \boldsymbol{e}_{2}
$$

where $\boldsymbol{r}_{P}$ defines the position of the point corresponding to $P$ in the reference cell $(0,0)$, while $\boldsymbol{e}_{1}$ and $\boldsymbol{e}_{2}$ are the lattice vectors. Any subsequently defined vectors will be denoted as bold, lower case letters, while matrices will be denoted as bold capital letters. Moreover, the notation ()$^{A}$ indicates components with respect to the vector basis $A$. Accordingly, the lattice vectors can be expressed as

$$
\begin{aligned}
& \boldsymbol{e}_{1}^{I}=\{R \cos \theta, R \sin \theta\}^{T} \\
& \boldsymbol{e}_{2}^{I}=\{-R \cos \theta, R \sin \theta\}^{T}
\end{aligned}
$$

Unit-Cell Periodicity via Bloch's Theorem. Wave propagation in structural lattices can be studied through the application of Bloch's theorem [10]. The displacement $\boldsymbol{w}$ of a point $P$ of the reference unit cell corresponding to a wave propagating at frequency $\omega$ can be expressed as:

$$
\boldsymbol{w}\left(\boldsymbol{r}_{P}\right)=\boldsymbol{w}_{P_{0}} e^{i \omega t-\boldsymbol{k} \cdot \boldsymbol{r}_{P}}
$$

where $\boldsymbol{w}_{P_{0}}$ is the wave amplitude, and $\boldsymbol{k}$ is the wave vector. According to Bloch's theorem, the displacement of the point corresponding to $P$ at location $\boldsymbol{\rho}_{p}\left(n_{1}, n_{2}\right)$ can be written in terms of the displacement of the reference unit cell as follows:

$$
\begin{aligned}
\boldsymbol{w}\left(\boldsymbol{\rho}_{P}\right) & =\boldsymbol{w}\left(\boldsymbol{r}_{P}\right) e^{\boldsymbol{k} \cdot\left(\boldsymbol{\rho}_{P}-\boldsymbol{r}_{P}\right)} \\
& =\boldsymbol{w}\left(\boldsymbol{r}_{P}\right) e^{n_{1} k_{1}+n_{2} k_{2}}
\end{aligned}
$$

where $k_{i}=\boldsymbol{k} \cdot \boldsymbol{e}_{i}$ with $i=1,2$. Bloch's theorem states that the proportionate change in wave amplitude occurring from cell to cell does not depend on the cell location within the periodic system, as such unit cell is merely a component of an infinite system. Accordingly, the wave propagation characteristics of the periodic assembly can be fully identified through the analysis of the reference unit cell, provided the system does not present any dissipation, as in the case of the lattice presented here. In Eqn. (4), $k_{i}=\delta_{i}+i \varepsilon_{i}(i=1,2)$ is a complex number, whose real part $\delta_{i}$ defines the amplitude attenuation as a wave propagates from one cell to the next, while the imaginary part $\varepsilon_{i}$ defines the change of phase across each cell, and it is therefore often called phase constant. In the analysis of wave propagation without amplitude attenuation, $\delta_{i}$ is typically set to 0 , while the phase constants $\varepsilon_{i}$ are varied to investigate their dependency upon frequency.

The space defined by the lattice vector basis $\mathcal{E}=\left(\boldsymbol{e}_{1}, \boldsymbol{e}_{2}\right)$ is denoted as the direct lattice. Given a direct lattice space, one may define a reciprocal lattice, which is described by the basis $\mathcal{B}=\left(\boldsymbol{b}_{1}, \boldsymbol{b}_{2}\right)$, whose basis vectors are given by:

$$
\boldsymbol{b}_{i} \cdot \boldsymbol{e}_{j}=\delta_{i j}
$$

where $\delta_{i j}$ is the Kronecker delta. The reciprocal lattice vectors for the considered periodic chiral assembly are given by:

$$
\begin{aligned}
& \boldsymbol{b}_{1}^{I}=\left(\frac{1}{2 R \cos \theta}, \frac{1}{2(R \sin \theta)}\right)^{T} \\
& \boldsymbol{b}_{2}^{I}=\left(-\frac{1}{2 R \cos \theta}, \frac{1}{2(R \sin \theta)}\right)^{T}
\end{aligned}
$$

In the reciprocal lattice, the wave vector $k=2 \pi \lambda$ can be expressed as:

$$
\boldsymbol{k}=k_{1} \boldsymbol{b}_{1}+k_{2} \boldsymbol{b}_{2}
$$

so that, according to the definition of reciprocal lattice given in Eqn. (6):

$$
\boldsymbol{k} \cdot \boldsymbol{e}_{1}=k_{1}
$$

While the direct lattice defines the spatial periodicity of the considered domain, the reciprocal lattice describes the periodicity of the frequency/wavenumber relation. This can be easily demonstrated by replacing $\lambda$, where $k=2 \pi \lambda$, with $\lambda^{\prime}=$ $\boldsymbol{\lambda}+m_{1} \boldsymbol{b}_{1}+m_{2} \boldsymbol{b}_{2}$ in Eqn. (4), with $m_{1}, m_{2}$ integers to obtain:

$$
\boldsymbol{w}\left(\boldsymbol{\rho}_{p}\right)=\boldsymbol{w}\left(\boldsymbol{r}_{p}\right) e^{n_{1} k_{1}^{\prime}+n_{2} k_{2}^{\prime}}
$$

where

$$
k_{i}^{\prime}=2 \pi \lambda^{\prime} \cdot \boldsymbol{e}_{i}=k_{i}+2 \pi m_{i}, \quad i=1,2
$$

Equation (10) indicates that the wavenumber in a 2-D lattice is a periodic function of the wave vector $\boldsymbol{k}$ in the reciprocal lattice. Hence, full representation of the dependency of the wave vector upon the frequency of wave propagation is obtained by investigating its variation over a single period. In a 2-D lattice, the spatial period corresponds to a region in the reciprocal lattice whose area equals the area of the reciprocal lattice's unit cell. The procedure for the identification of the area corresponding to the spatial period, also known as first Brillouin zone, is described in [11]. The chosen first Brillouin zone for the chiral structural lattice is presented in the following section. 
Analysis of Free-Wave Motion: Governing Equations and Boundary Conditions. The behavior of the unit cell of a periodic structural assembly can be conveniently described through a discretized equation of motion in matrix form and by defining the cell's interaction with its neighbors (Fig. 2). The unit cell depicted in Fig. 3, is modeled as an assembly of rigidly connected beams, whose behavior is described by axial and transverse degrees of freedom (dof), as well as rotational dof's according to the Timoshenko beam model [12]. Application of standard finite-element procedures yields the unit cell's equations of motion, which can be expressed as:

$$
\left(\boldsymbol{K}-\omega^{2} \boldsymbol{M}\right) \boldsymbol{u}=\boldsymbol{f}
$$

where $\omega$ is the frequency of wave propagation, $\boldsymbol{K}$ and $\boldsymbol{M}$ are the assembled mass and stiffness matrices for the cell, while $\boldsymbol{u}, \boldsymbol{f}$ are respectively the vectors containing generalized nodal displacements and forces of interaction of the cell with its neighbors. Such generalized nodal vectors are defined as follows:

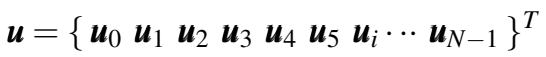

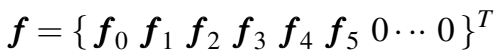

In Eqn. (12) the subscripts $0,1, \cdots, 5$ follow the notation described in Fig. 3, while subscript $i$ denotes the degrees of freedom of nodes that are internal to the cell, which is discretized by a total of $N$ nodes. Eq. (12) is also based on the assumption that no external forces are applied, and that only the interaction forces with neighboring cells appear in the cell's equation of motion. The absence of external forces on the internal nodes facilitates the condensation of the internal degrees of freedom, which yields a significant reduction in the size of the problem.

According to Bloch's theorem, periodic boundary conditions relate the cell's generalized displacements, in addition to equilibrium conditions which can be applied to the generalized forces [10]:

$$
\begin{aligned}
& \boldsymbol{u}_{3}=e^{k_{1}} \boldsymbol{u}_{0}, \\
& \boldsymbol{u}_{4}=e^{k_{1}+k_{2}} \boldsymbol{u}_{1} \\
& \boldsymbol{u}_{5}=e^{k_{2}} \boldsymbol{u}_{2}
\end{aligned}
$$

and

$$
\begin{aligned}
& \boldsymbol{f}_{3}=-e^{k_{1}} \boldsymbol{f}_{0}, \\
& \boldsymbol{f}_{4}=-e^{k_{2}} \boldsymbol{f}_{1} \\
& \boldsymbol{f}_{5}=-e^{k_{1}+k_{2}} \boldsymbol{f}_{2}
\end{aligned}
$$

Equations (13) and (14) can be rewritten in matrix form as follows:

$$
\begin{aligned}
& \boldsymbol{u}=\boldsymbol{A} \boldsymbol{u}_{r}, \\
& \boldsymbol{f}=\boldsymbol{B} \boldsymbol{f}_{r}
\end{aligned}
$$

where $\boldsymbol{u}_{r}=\left\{\begin{array}{llll}\boldsymbol{u}_{0} & \boldsymbol{u}_{1} & \boldsymbol{u}_{2} & \boldsymbol{u}_{i}\end{array}\right\}^{T}$. Substituting eq. (13) into eq. (11), pre-multiplying the resulting equations for $\mathbf{A}^{H}$, with ()$^{H}$ denoting a complex conjugate transpose, and assuming $\boldsymbol{f}_{i}=0$ gives:

$$
\left[\boldsymbol{K}_{r}\left(k_{1}, k_{2}\right)-\omega^{2} \boldsymbol{M}_{r}\left(k_{1}, k_{2}\right)\right] \boldsymbol{u}_{r}=\mathbf{0}
$$

where $\boldsymbol{K}_{r}\left(k_{1}, k_{2}\right), \boldsymbol{M}_{r}\left(k_{1}, k_{2}\right)$ are reduced stiffness and mass matrices. Equation (17) is an eigenvalue problem whose solution defines the dispersion characteristics of the lattice. The solution requires imposing two of the three unknowns $k_{1}, k_{2}, \omega$, and solving for the third. Typically, wave motion without attenuation is investigated. For this reason, $k_{1}, k_{2}$ are generally imposed as imaginary numbers $k_{1}=i \varepsilon_{1}, k_{2}=i \varepsilon_{2}$ which vary within the first Brillouin zone. The solution of the eigenvalue problem yields the frequency of wave propagation corresponding to the assigned pair $k_{1}, k_{2}$. The complete solution obtained for varying $k_{1}, k_{2}$ is a surface $\omega=\omega\left(k_{1}, k_{2}\right)$ denoted as phase constant surface or dispersion surface. The number of surfaces obtained corresponds to the dimension of the eigenvalue problem in eq. (17). Each surface describes the wavenumber/frequency relation for the corresponding mode of wave propagation in the considered medium. The computational cost to obtain dispersion diagrams can be significantly reduced by exploiting the symmetry of the first Brillouin zone, which is related to the symmetry of the unit cell. As a result, smaller regions of variation for the phase constants can be identified, which in most cases capture maxima and minima of the dispersion surfaces. Such regions are denoted as irreducible Brillouin zones [11]. In addition, information regarding propagation in specific directions, or on the presence of frequency gaps between consecutive phase constant surfaces can be obtained by letting the wave vector vary only along the contour of the irreducible zone. Examples of irreducible zones and various possible representations of the dispersion diagrams are presented in the next section.

Phase and group velocities. The dispersion surfaces are used to estimate the variation of the phase and group velocity in terms of frequency and direction of wave propagation. The phase velocity in a direction defined by the wave vector:

$$
\begin{aligned}
& \boldsymbol{k}=k_{1} \boldsymbol{b}_{1}+k_{2} \boldsymbol{b}_{2} \\
& \boldsymbol{k}=\xi_{1} \boldsymbol{i}_{1}+\xi_{2} \boldsymbol{i}_{2}
\end{aligned}
$$


and at frequency $\omega$ is given by:

$$
c_{p h}=\frac{\omega}{k} u
$$

where $k=|\boldsymbol{k}|$ and $\boldsymbol{u}$ is a unit vector in the direction of the wave vector $(\boldsymbol{u}=\boldsymbol{k} / k)$. The phase velocity is evaluated by selecting the wavenumbers corresponding to the dispersion surfaces at the desired frequency value. The angular range spanned by the corresponding set of wavenumbers is then computed to obtain the variation of the phase velocity in terms of the direction of wave propagation. For a non-dispersive, homogeneous medium, this operation yields a circle whose radius is independent of frequency. The same information is commonly presented in the form of slowness curves, which are plots of the inverse of the phase velocity [13]. In the following examples, however, it is chosen to directly represent variations of velocity in terms of direction of wave propagation and frequency. Such variations may also be directly observed from the time domain simulation of wave propagation within a cellular domain.

Important indications regarding the wave characteristics of a lattice are also provided by the group velocity, which can be estimated from the dispersion surfaces. The group velocity in the direct lattice space can be expressed as:

$$
\boldsymbol{c}_{g}^{\mathcal{E}}=\left(\frac{\partial \omega}{\partial k_{1}}, \frac{\partial \omega}{\partial k_{2}}\right)^{T}
$$

which can be transformed into components in the physical space $I$ through the following vector manipulation:

$$
\begin{aligned}
\boldsymbol{c}_{g}^{I} & =\left(\begin{array}{l}
\boldsymbol{e}_{1} \cdot \boldsymbol{i}_{1}, \boldsymbol{e}_{2} \cdot \boldsymbol{i}_{1} \\
\boldsymbol{e}_{1} \cdot \boldsymbol{i}_{2}, \boldsymbol{e}_{2} \cdot \boldsymbol{i}_{2}
\end{array}\right) \boldsymbol{c}_{g}^{\mathcal{E}} \\
\boldsymbol{c}_{g}^{I} & =\boldsymbol{E} \boldsymbol{c}_{g}^{\mathcal{E}}
\end{aligned}
$$

Matrix $\boldsymbol{E}$ contains the components of the direct lattice basis with respect to the physical reference system $I$. The group velocity defines the direction of energy flow within the structure, and therefore identifies preferential or forbidden directions for wave propagation [5]. In addition, the dispersive nature of the medium can also be highlighted from a discrepancy between group and phase velocity. Such a discrepancy can occur both for increasing frequency and, more interestingly, along different directions. At a given frequency, the lattice may behave in a dispersive fashion in a range of directions, while being non-dispersive in others. The evaluation of group and phase velocities allows determining the dispersive behavior of the lattice, indicated by the frequency dependency of the phase velocity, and the anisotropy of the domain in the wave propagation plane. Dispersion surfaces, phase and group velocities for a chiral lattice are discussed in the next section.

\section{RESULTS AND DISCUSSION Dispersion surfaces.}

The contours of the first two branches of the dispersion surfaces, or the first two eigenmodes from Eqn. (17), for a chiral lattice defined by $L=0.1, L / R=0.90, t_{b}=t_{c}=1 \mathrm{~mm}$ and outof-plane width of $1 \mathrm{~mm}$, are depicted Fig. 4 and 5. Such con-

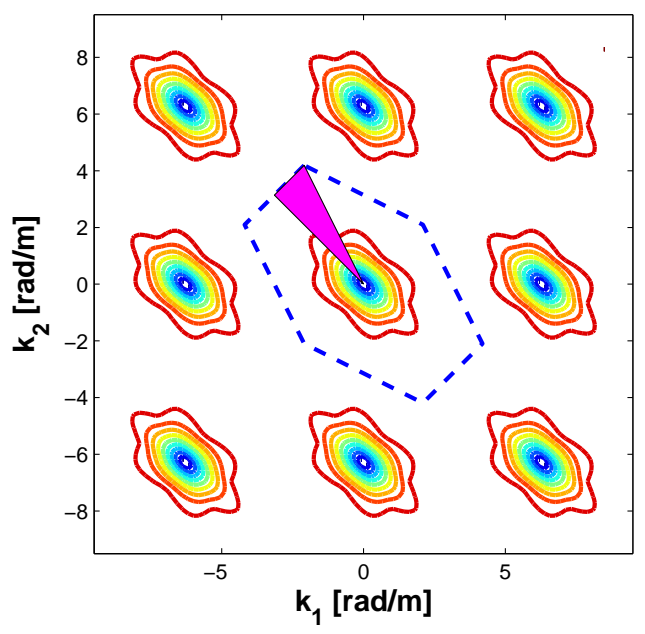

(a) I mode

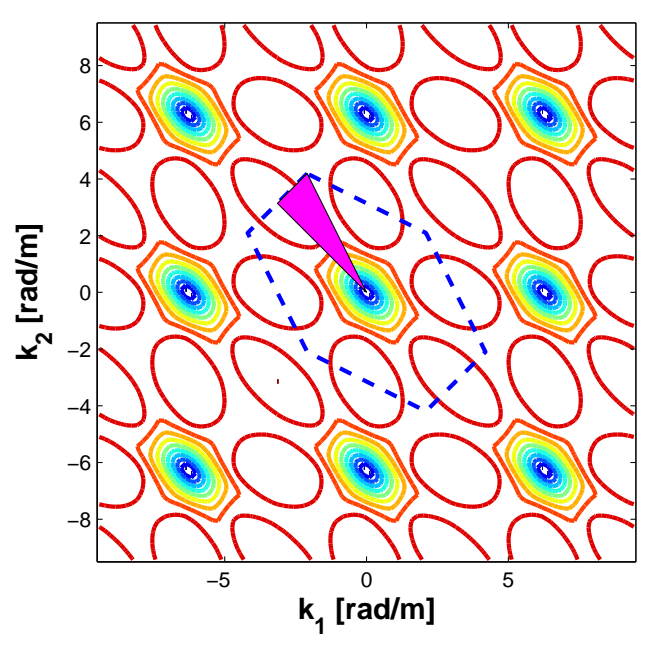

(b) II mode

Figure 4. DISPERSION CONTOURS FOR A CHIRAL LATTICE WITH $L / R=90$ - I AND I| EIGENMODE

tours show the periodicity of the dispersion relations, which is further confirmed by superimposing the boundaries of the first Brillouin zone to the underlying contour lines. Moreover, contour plots corresponding to the first and second eigenmodes show 


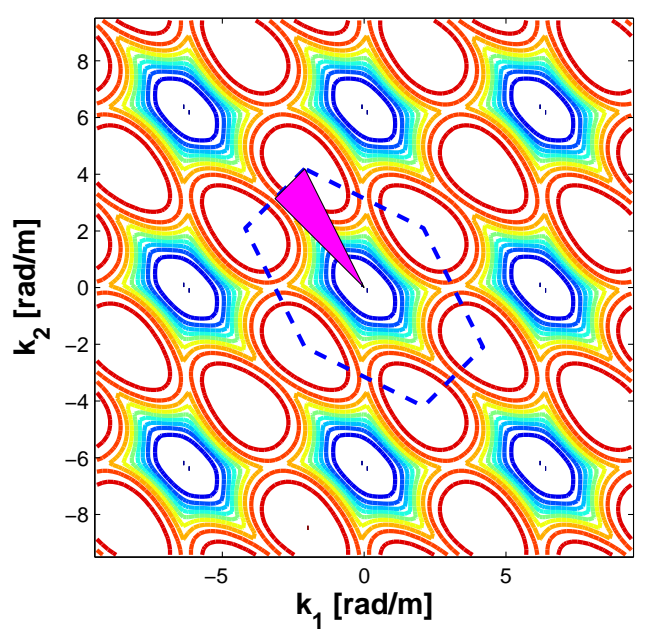

(a) III mode

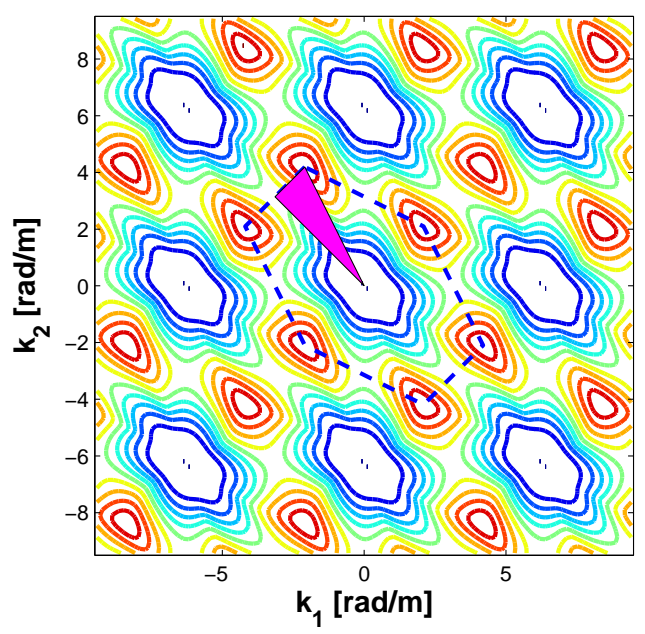

(b) IV mode

Figure 5. DISPERSION CONTOURS FOR A CHIRAL LATTICE WITH $L / R=90-$ III AND IV EIGENMODE

non directional behavior at low frequencies, while directionality is encountered at higher frequencies, as indicated by concentric lines, which vary from a lobed to a circular pattern, moving from the outside inward. On the contrary, eigenmodes III and IV are strongly directional over the considered frequency range. The analysis of dispersion surfaces suggests that the chiral lattice features isotropic behavior in the low frequency limit, as for static analyses by [8], while directional effects are dominant at higher frequencies.

\section{Band structure}

The symmetry of the first Brillouin zone can be exploited to limit the variation of the wave vector along the irreducible zone, which can be defined as the smallest area allowing full representation of the $\omega=\omega\left(k_{1}, k_{2}\right)$ variation. The irreducible zone in

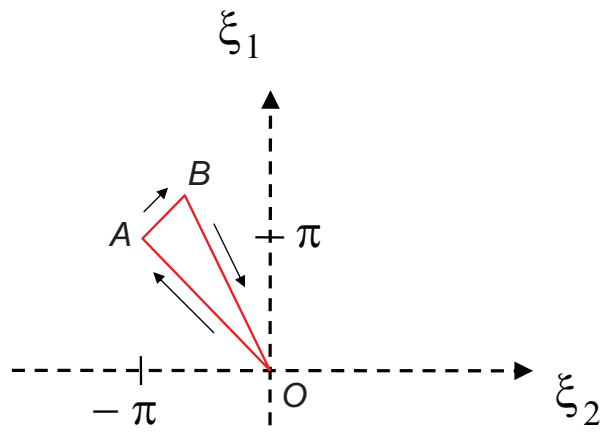

(a) Brillouin irreducible zone

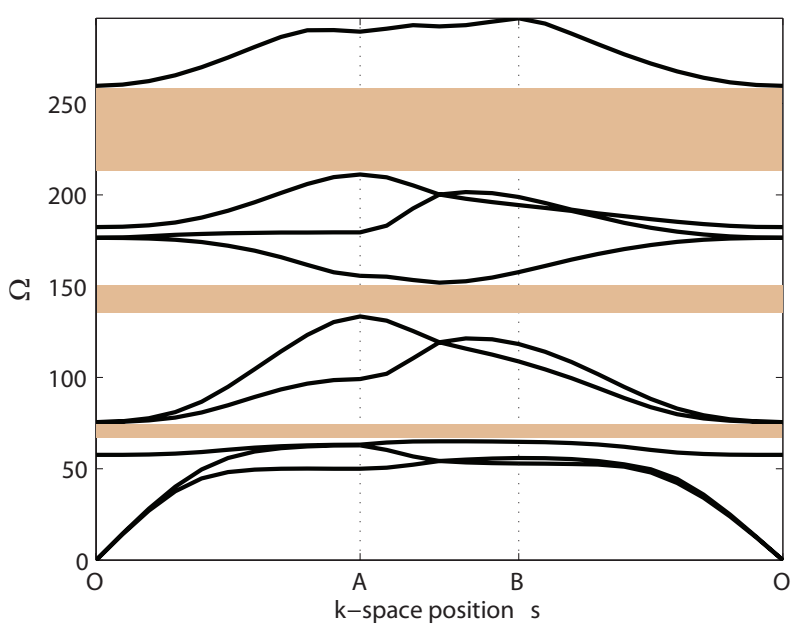

(b) Band structure - Lowest nine modes

Figure 6. BAND STRUCTURE FOR A CHIRAL LATTICE WITH $L / R=$ 0.9 .

the wavenumber space $k_{1}, k_{2}$ is shown in Fig. 6.a, while the dispersion curves calculated along the perimeter of the irreducible zone are shown in Fig. 6.b, where the frequency axis is normalized with respect to the flexural resonance of a of ligament of length $L$ (Fig. 1), so that:

$$
\Omega=\omega \frac{L^{2}}{\pi^{2}} \sqrt{\frac{\rho t}{E I}}
$$

with $\omega$ denoting the frequency (or the eigenvalue from Eqn. 17) corresponding to the assigned wavenumber. $E, \rho$ and $I$ denote 
Young's modulus, density of constituent material and second moment of inertia of the lattice frame-elements respectively.

Fig. 6.b depicts dispersion relations as obtained using the irreducible zone. The first two propagation modes feature a constant slope at low frequency, and therefore suggest that the wave velocity is preserved along all directions, in accordance with phase constant surfaces. Fig. 6.b also shows the presence of peculiar wave phenomena such as mode veering and bandgaps [4, 14]. Veering consists of two branches coming infinitely close without crossing or touching, and it is a common phenomenon found in the solution of eigenvalue problems containing weakly coupled systems $[14,15]$. The curves corresponding to first and second propagation modes in Fig. 6 (locations $A-B)$ appear confined to a narrow frequency range and with limited separation, yielding veering phenomena which are usually encountered at higher frequencies. The configuration defined by $L / R=0.9$ also features a unique distribution of band gaps even for relatively low frequency values. Three band gaps are clearly observed within the first nine propagation modes (Fig. 6). The presence of significant stop bands at low frequencies suggests the considered configuration as an effective vibration filter, compared to hexagonal honeycombs [4], which present stop bands at much higher normalized frequencies. The eigenmodes corresponding to the first, second, third and fifth branches of the band structure (Eqn. 17) are depicted in Fig. 7.

\section{Phase and Group Velocities}

Phase velocities are evaluated to highlight the non-isotropic propagation behavior of the lattices, as well as their dispersive characteristics. In what follows, results are presented for velocities corresponding to the first two surfaces of the dispersion diagrams (Fig. 4). For convenience, and in analogy with the behavior of a homogeneous solid, the modes corresponding to the lowest two branches are here denoted as shear (S-mode) and longitudinal (P-mode), respectively. The angular variation of the Smode and of the P-mode phase velocities is shown respectively in fig.s 8.a and 8.b. The chiral honeycomb behaves as an isotropic solid at low frequencies, as indicated by the corresponding circular velocity curves. It is also interesting to observe that the low-frequency behavior of the lattice appears as non-dispersive as the velocity curves at different frequencies overlap almost exactly. The non-isotropic behavior of the lattice at higher frequencies appears evident from the curve at $\Omega=50$ which recalls the six-fold, hexagonal symmetry of the lattice. In addition, the low frequency phase velocities of mode $\mathrm{S}$ and $\mathrm{P}$ are very close to each other, in accordance with the observations made about the first two branches of the dispersion curves along the contour of the first Brillouin zone. The same observations can be put forth for the group velocities shown in Fig. 9.
I MODE

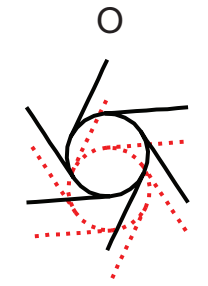

A

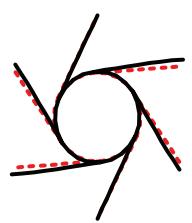

B
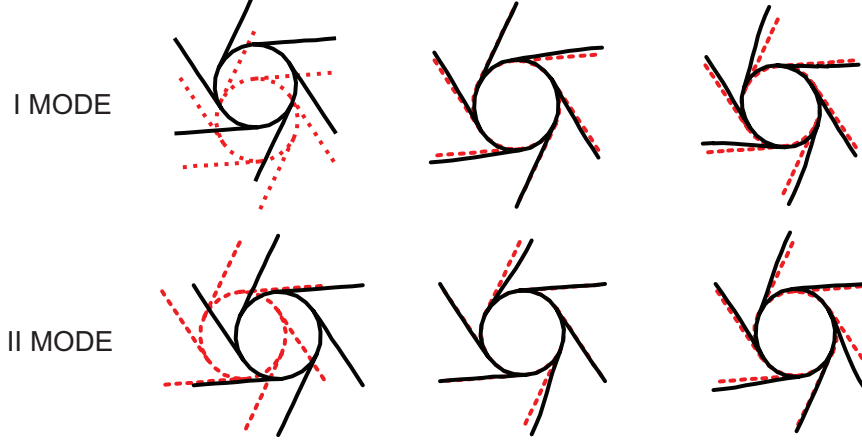

III MODE
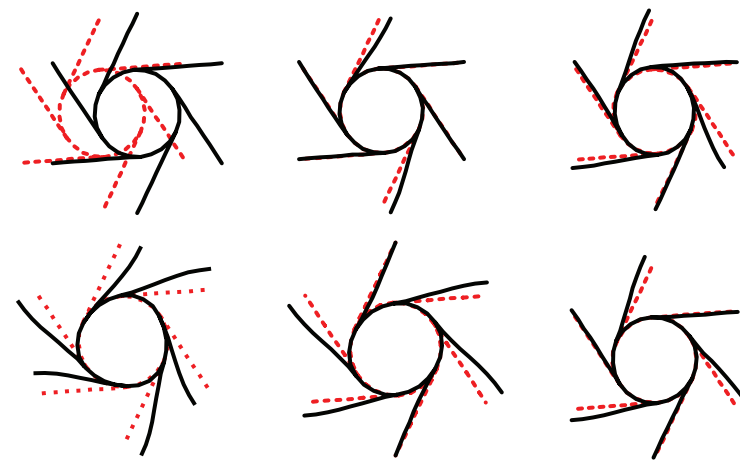

V MODE
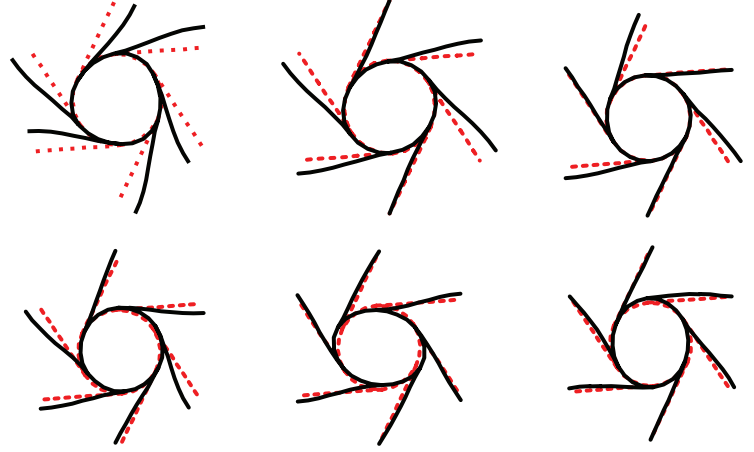

Figure 7. EIGENMODES CORRESPONDING TO DIFFERENT COMBINATIONS OF $k$ AND $\omega$ AS FOR Eqn. 17.

\section{Influence of Geometric Parameters.}

The character of the band structure previously analyzed suggests that the chiral lattice features several stop bands of considerable width even at low frequency. Within the first ten modes, in fact, three band gaps are observed, one of which spans over a large portion of the considered frequency range. It is interesting to study the effect of the characteristic parameters of the unit cell on the band gap behavior. More specifically, we want to investigate how geometric quantities defining the unit cell affect the location and width of the band gaps, compared to the baseline configuration given by $L / R=0.90$. Such analysis is of great interest to design configurations which maximize the filtering properties of the proposed lattice.

The first parameter considered for this survey is the ratio $L / R$, which was previously defined. Several configurations for values of $L / R$ between 0.75 and 0.98 are considered. The variation of bandgap width and frequency range is shown in Fig. 10. The density of band-gap regions is maximum in the region corresponding to values of $L / R$ between 0.8 and 0.9 . The three band gaps presented in Fig. 6 can also be observed in Fig. 10 corresponding to $L / R=0.9$.

The second geometric parameter to be investigated is the wall thickness distribution within the unit cell. In particular, the 


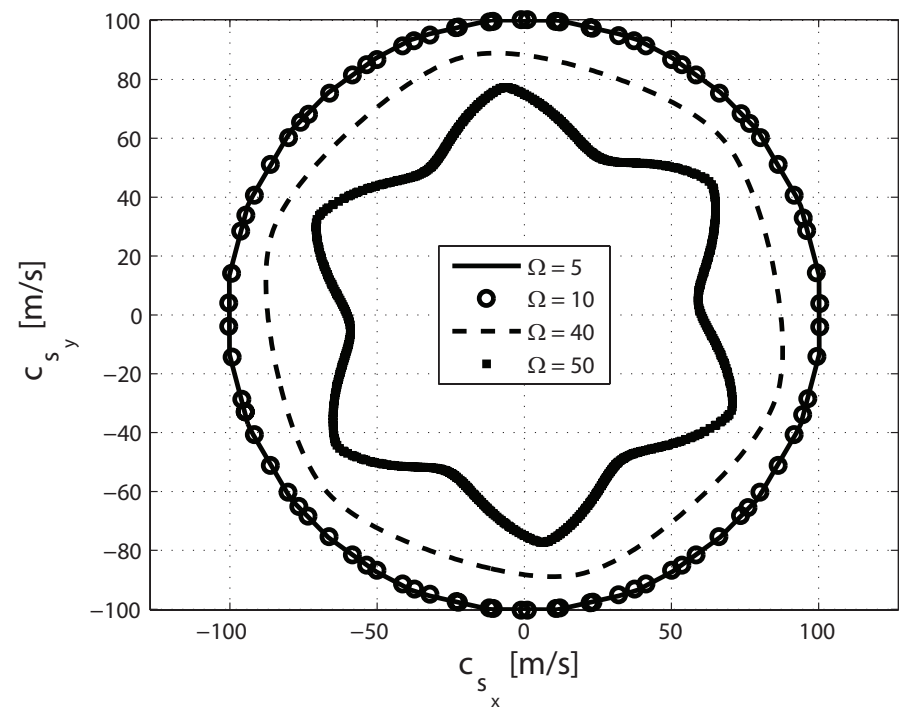

(a) S- mode

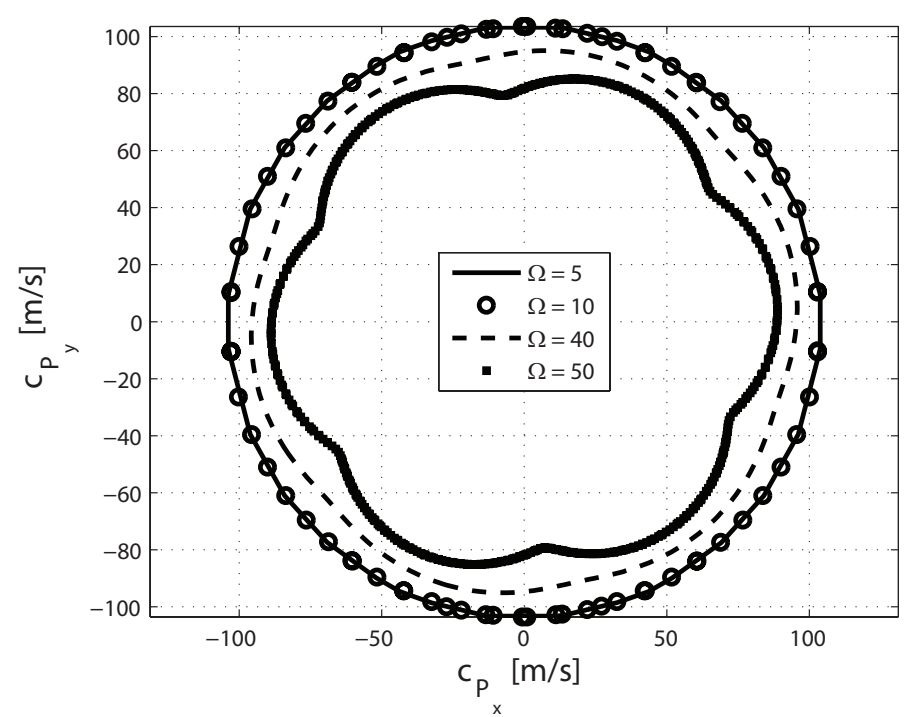

(b) P- mode

Figure 8. PHASE VELOCITIES CORRESPONDING TO DIFFERENT WAVE FREQUENCIES

effects on band gap distribution by the thickness of the circular elements are analyzed. The mode shapes depicted in Fig. 7 suggest that the deformation of the circles does not follow a clear pattern from that of the ligaments; therefore, the deformation of nodes is an important aspect to be considered as a wave propagation mechanism. The analysis is first carried out by varying $t_{c}$ while keeping the thickness of the ligaments $t_{b}$ constant as shown in Fig. 3. This produces the band gap distribution of Fig. 11, where $K=t_{c} / t$, where $t$ is the value used for previous analyses (e.g. $1 \mathrm{~mm}$ ). A pattern is observed whereby the lowest bang gap

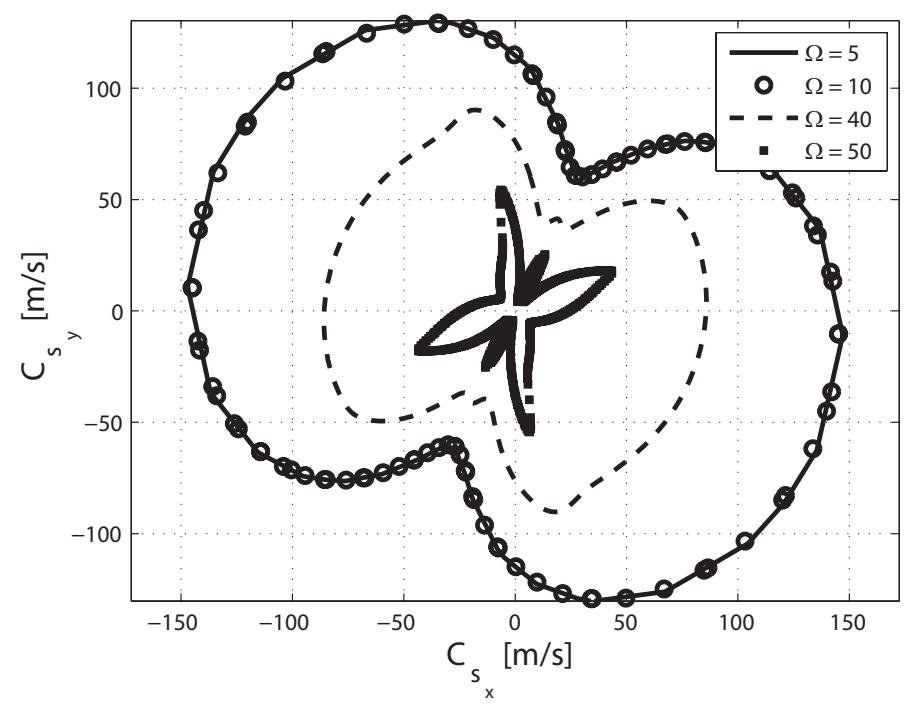

(a) S- mode

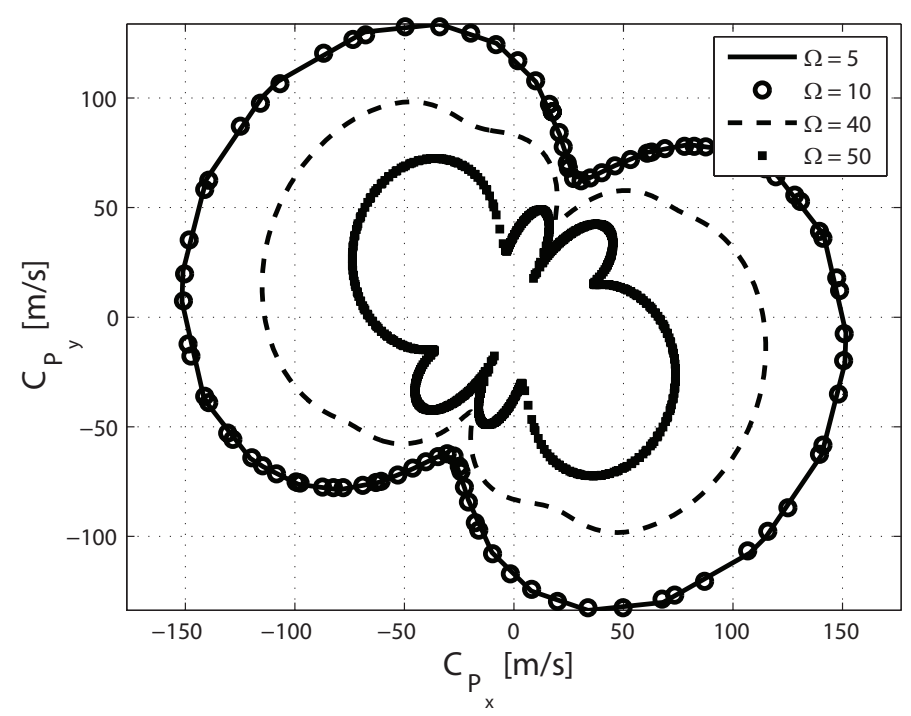

(b) P- mode

Figure 9. GROUP VELOCITIES FOR VARYING WAVE FREQUENCIES.

is encountered at an almost constant frequency; the second and third band gap move towards higher frequencies as the value of $K$ increases. In other words, stiffer circles may give rise to almost gap-free, low-frequency range. This may suggest that the first band gap, whose location is invariant with $K$, is mainly related to the deformation of the straight links.

It is of interest to analyze the influence of the wall thickness $t_{c}$ and $t_{b}$ independently from the mass of the unit cell, which is maintained constant. This is done by letting $t_{c}=K t$ and $t_{b}=1 / K t$. Results shown in Fig. 12 indicate strong influence 


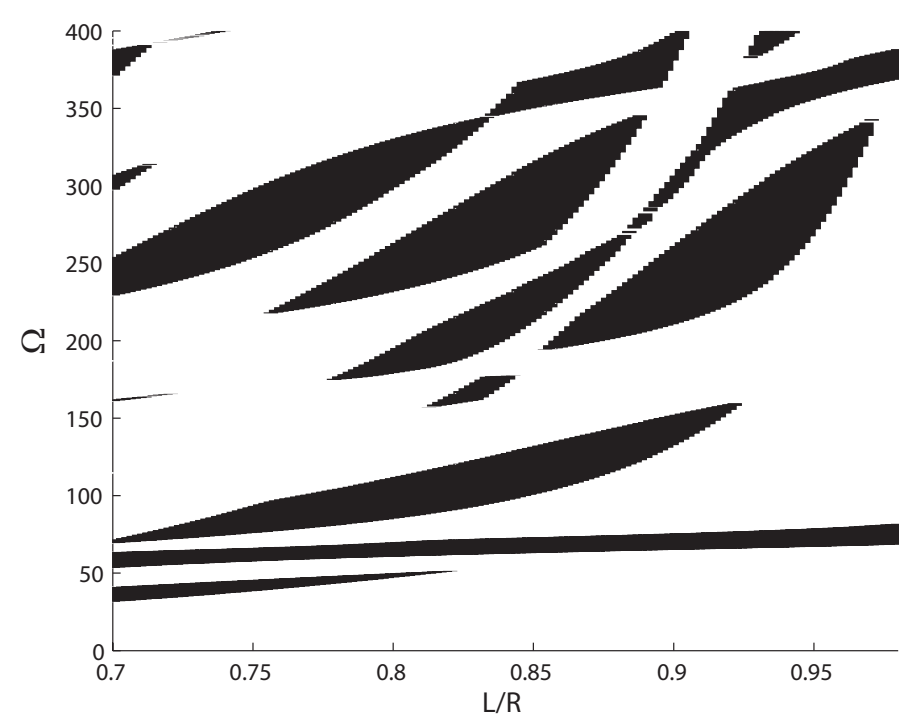

Figure 10. BAND GAP DISTRIBUTION RESULTING FROM DIFFERENT $L / R$ RATIOS.

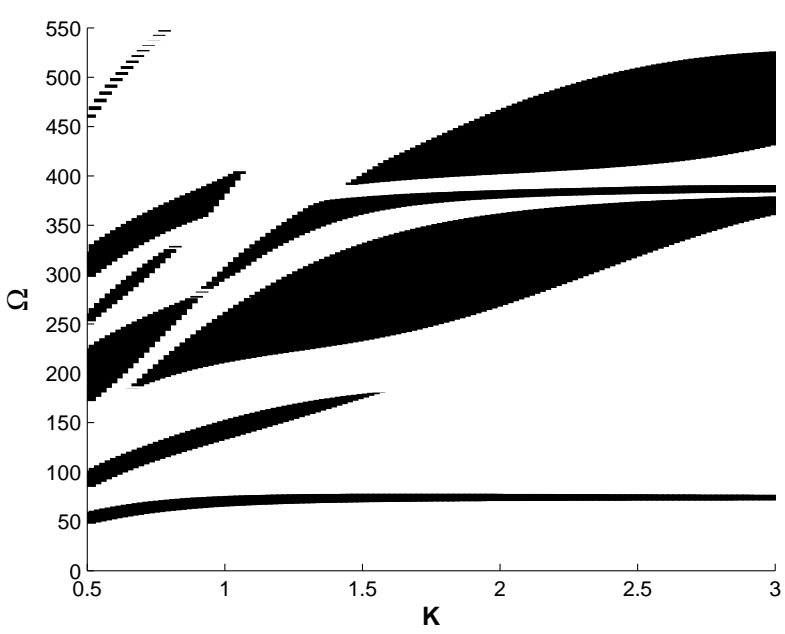

Figure 11. BAND GAP DISTRIBUTION OBTAINED VARYING $t_{c}$.

of varying $t_{c}$ and $t_{b}$ simultaneously upon the wave propagation behavior of the chiral structure. Fig. 12 reveals that a high concentration of band gaps occurs around particular combinations of $K$ and $\Omega$. Accordingly the band gaps shift towards higher frequency values as the difference in thickness between the nodes and ligaments increases.

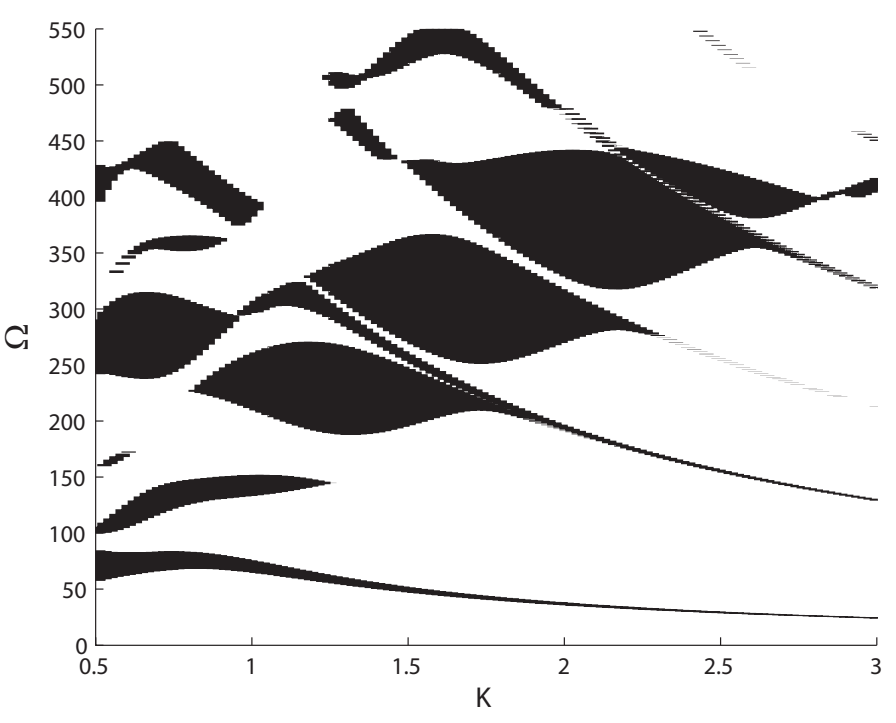

Figure 12. BAND GAP DISTRIBUTION OBTAINED VARYING $t_{c}$ AND $t_{b}$.

\section{CONCLUSION \\ REFERENCES}

[1] Gibbson, L., and Ashby, F., 2001. Cellular Solids: Structures and Properties. Cambridge University Press, Cambridge, UK.

[2] Langley, R., 1996. "Response of two-dimensional periodic structures to point harmonic forcing". Journal of Sound and Vibration, 197(4), pp. 447-469.

[3] Sigmund, O., and Jensen, J., 2003. "Systematic design of phononic band-gap materials and structures by topology optimization". Phylosophical Transactions of the Royal Society London, Series A (Mathematical, Physical and Engineering Sciences, 361(1806), pp. 1001-1019.

[4] Srikantha Phani, A., Woodhouse, J., and Fleck, N., 2006. "Wave propagation in two-dimensional periodic lattices". Journal of the Acoustical Society of America, 119(4), pp. $1995-2005$.

[5] Ruzzene, M., Scarpa, F., and Soranna, F., 2003. "Wave beaming effects in two-dimensional cellular structures". Smart Materials and Structures, 12(3), pp. 363 - 372.

[6] Jeong, S. M., and Ruzzene, M., 2004. "Analysis of vibration and wave propagation in cylindrical grid-like structures". Shock and Vibration, 11(3-4), pp. 311 - 331.

[7] Lakes, R. S., 1991. "Deformation mechanisms of negative poisson's ratio materials: structural aspects". Journal of Material Science, 26, pp. 2287 - 2292.

[8] Prall, D., and Lakes, R., 1997. "Properties of a chiral honeycomb with a poisson's ratio of - 1". International Journal of Mechanical Sciences, 39(3), pp. 305 - 314.

[9] Lakes, R., 2001. "Elastic and viscoelastic behavior of chiral 
materials". International Journal of Mechanical Sciences, 43(7), pp. 1579 - 1589.

[10] Mead, O., 1973. "A general theory of harmonic wave propagation in linear periodic systems with multiple coupling". Journal of Sound and Vibration, 27, pp. $235-260$.

[11] Brillouin, L., 1953. Wave Propagation in Periodic Structures. Dover, New York, NY.

[12] Cook, R. D., Malkus, D. S., Plesha, M. E., and Witt, R. J., 2001. Concepts and Applications of Finite Element Analysis, $4^{\text {th }}$ ed., Vol. 2. Wiley, New York, NY.

[13] Auld, B. A., 1990. Acoustic Fields and Waves in Solids, $2^{\text {nd }}$ ed. Krieger Publ. Co., Malabar, FL.

[14] Perkins, N. C., and Mote, C. D. J., 1986. "Comments on curve veering in eigenvalue problems.”. Journal of Sound and Vibration, 106(3), pp. $451-463$.

[15] Nair, P. S., and Durvasula, S., 1973. "On quasidegeneracies in plate vibration problems.". International Journal of Mechanical Sciences, 15(12), pp. 975 - 986. 